\title{
ArcheoSciences
}

Revue d'archéométrie

$30 \mid 2006$

Varia

\section{Production expérimentale de laiton par cémentation en creuset ouvert, avec du minerai de zinc, selon les recettes médiévales et modernes}

Experimental production of brass by cementation, in opened crucibles, with zinc ore, according to the medieval and modern receipts

\section{Aurore Doridot, Luc Robbiola et Florian Tereygeol}

\section{OpenEdition}

Journals

Édition électronique

URL : http://journals.openedition.org/archeosciences/107

DOI : 10.4000/archeosciences. 107

ISBN : 978-2-7535-1595-6

ISSN : 2104-3728

Éditeur

Presses universitaires de Rennes

\section{Édition imprimée}

Date de publication : 31 décembre 2006

Pagination : $15-24$

ISBN : 978-2-7535-0456-1

ISSN : $1960-1360$

Référence électronique

Aurore Doridot, Luc Robbiola et Florian Tereygeol, « Production expérimentale de laiton par cémentation en creuset ouvert, avec du minerai de zinc, selon les recettes médiévales et modernes », ArcheoSciences [En ligne], 30 | 2006, document 2, mis en ligne le 31 décembre 2008, consulté le 19 avril 2019. URL : http://journals.openedition.org/archeosciences/107 ; DOI : 10.4000/ archeosciences. 107 


\title{
Production expérimentale de laiton par cémentation en creuset ouvert, avec du minerai de zinc, selon les recettes médiévales et modernes
}

\author{
Aurore Doridot*, Luc Robbiola**, Florian TÉREYGeol ${ }^{* * *}$
}

\begin{abstract}
Résumé : Actuellement deux sources permettent d'apprécier le mode de fabrication du laiton par cémentation pour les périodes anciennes et tout particulièrement pour le Moyen Âge. Il s'agit des textes et des découvertes archéologiques. Les fouilles archéologiques présentent l'utilisation de deux types de creusets, certains restent ouverts alors que d'autres sont complètement obstrués. Les textes, eux, ne mentionnent que l'usage de creusets ouverts. D'autre part d'après de nombreux traités de chimie moderne, l'emploi du creusets ouverts pour la fabrication de laiton par cémentation ne permet pas d'obtenir de concentrations en zinc supérieures à 28 voire $22 \%$ massique. Afin de répondre aux nombreuses questions que soulève l'étude des données archéologiques et textuelles, une approche expérimentale a été réalisée à Melle. Quatre essais ont été effectués en variant la nature du minerai de zinc. Les résultats confirment la formation de laiton à des taux moyens compris entre 23 et $30 \%$ massique de zinc. Les données analytiques et structurales, notamment les teneurs en zinc et les hétérogénéités de composition, ont permis de conclure à la pertinence des recettes médiévale et modernes.
\end{abstract}

\begin{abstract}
Today two main sources could be used to understanding brass making process in medieval ages: writing sources and archaeological ones. In order to answer to the numerous questions rising up by historical and archaeological studies, we attempt an experimental approach at Melle. Excavations put in light two kinds of crucible: uncovered ones and closed ones (by lay or a lid). Reading modern chemical treaties (from the $19^{\text {th }}$ to the $20^{\text {th }}$ century), it appears that the use of uncovered crucible can not produce brass with high rate of zinc (never more than $28 \%$ weight). What quality of brass could we produce following medieval and moderns recipes? Trying to answer, four attempts were lead using zinc ore (calamine) as cement. Results prove a good production of brass between 23 and 30\% weight. Chemical and structural data confirm the accuracy of medieval and modern recipes.
\end{abstract}

Mots clés : Cémentation, calamine, laiton, expérimentation, creuset, bas-foyer.

Key words: Cementation, calamine, brass, archaeological experiment, crucible, furnace.

\section{INTRODUCTION}

Connue et employée dès l'Antiquité, la fabrication du laiton par cémentation du cuivre est un procédé qui fait appel, entre autre, à l'utilisation de composés de zinc. L'un de ces composés communément appelé calamine se trouve men- tionné dès le I ${ }^{\text {er }}$ siècle de notre ère dans l'Histoire Naturelle de Pline l'Ancien (Le Bonniec, 2003). Il s'agit d'un minerai de zinc connu sous le terme de cadmea. Les écrits de Pline l'Ancien ne nous renseignent pas sur la préparation et le déroulement des opérations. Il faut attendre le texte de Théophile, auteur du XII ${ }^{\mathrm{e}}$ siècle, pour disposer d'un cha-

* Doctorante, UMR 8589 LAMOP, Université Paris 1, Panthéon-Sorbonne, Paris et ENSCP Laboratoire de Métallurgie Structurale, Paris.

(doridotaurore@yahoo.fr)

** Ingénieur de Recherche CNRS, ENSCP Laboratoire de Métallurgie Structurale, Paris. (luc-robbiola@enscp.jussieu.fr)

*** Chargé de Recherche CNRS, UMR 5060 Institut de Recherche sur les Archéomatériaux, Belfort et UMR 9956 Laboratoire Pierre Süe, CEA-CNRS,

CEA, Saclay. (tereygeol@drecam.cea.fr) 
pitre sur le procédé d'élaboration du laiton par cémentation (Escalopier, 1977; Hawthorne et Smith, 1979). Au $\mathrm{XVI}^{\mathrm{e}}$ siècle, les auteurs des grands traités de métallurgie et de minéralogie comme Giorgius Agricola dans De Natura Fossilium, Vanoccio Biringuccio dans De la Pirotechnia et Lazarus Ercker dans son Beschreibung Allerfürnemisten Mineralischen Ertzt unnd Bergkwercks arten décrivent ce procédé en y ajoutant chacun une note très personnelle. À partir du XviII ${ }^{e}$ siècle, le laiton se fabrique selon deux méthodes. La technique par cémentation est conservée (Anonyme, 1747; Macquer, 1777) et l'autre méthode utilise la fusion du cuivre avec du zinc métallique (Henckel, 1756). Certains auteurs vont jusqu’à conseiller de mixer les deux techniques pour un meilleur résultat (Dumas, 1833). Or dès 1833, M. Dumas présage, à court terme, de la disparition du procédé par cémentation puisqu'il écrit à ce moment : «Il est probable que les procédés basés sur l'emploi des oxides, quelle qu'en soit l'origine, disparaîtront partout, pour faire place au procédé direct. " (Dumas, 1833). Effectivement, en 1873, A. D. Wurtz écrit dans son Dictionnaire de chimie pure et appliquée que « L'ancien procédé de cémentation est universellement remplacé par l'alliage direct des deux métaux. " (Wurtz, 1873). Dans le cadre des études de recréation du procédé ancien, tous les essais ont été conduits en laboratoire, la plupart utilisant de l'oxyde de zinc et non un minerai de zinc. Une synthèse de ces données a été faite par J.-M. Welter en 2003 dans The zinc content of brass: a chronological indicator?

D'un point de vue archéologique, les exemples d'objets reconnus comme ayant servi à la cémentation du cuivre sont relativement peu nombreux. Ces découvertes, encore marginales, sont pour la plupart datées de l'époque romaine (Picon, 1995; Bayley, 1998; Rehren, 1999; Desbat et al., 2000). Elles présentent des contenants de tailles très différentes. Les séries trouvées en France (Lyon, Autun et Alésia) mesurent environ cinquante centimètres de haut et peuvent contenir jusqu'à quarante litres, cela présuppose une production de grande ampleur (Picon, 1995; Desbat et al., 2000). Quant aux séries anglaise et allemande, il s'agit de petits spécimens ne dépassant pas les cinq centimètres de haut et ayant une contenance très réduite de deux à cinq centilitres (Bayley, 1998; Rehren, 1999). D’autres travaux présentent la découverte de creusets ouverts ayant servi à la fabrication du laiton par cémentation et datant de la période carolingienne (Rehren, 1999; Krabath et al., 1999). Ils proviennent d'Allemagne et plus particulièrement de Dortmund, Soest et Schwerte. De morphologie très différente des spécimens d'époque romaine, ils ont une hauteur de quatre à onze centimètres et une contenance d'environ deux à cinquante centilitres. Un important travail de compilation de ces sources a été réalisé par Thilo Rehren (Rehren, 2003). À ceci, il faut ajouter les creusets de Zwickau (Allemagne) datés de la dernière moitié du XvI ${ }^{\mathrm{e}}$ siècle (Martinón-Torres et Rehren, 2002). D'une hauteur de quarante centimètres, ils pouvaient contenir jusqu'à douze litres de matières. Leur particularité repose sur la présence d'un couvercle ayant une ouverture centrée de deux centimètres de diamètre. En se référant aux sources écrites (Théophile, Agricola, Ercker, Henckel, Anonyme et Macquer), seuls les creusets ouverts semblent avoir été utilisés. En effet, les descriptions données par les différents textes précisent que ces contenants doivent être surveillés afin de distinguer le laiton formé, ou encore les fumées qui s'en échappent, et ainsi mettre fin opportunément à l'opération. De ce fait, ces écrits laissent supposer que les creusets utilisés restaient ouverts c'est-à-dire ne présentaient pas de couvercle permettant de les obstruer durablement. Seul le texte de Biringuccio ne fournit pas d'informations suffisamment précises pour échafauder une quelconque hypothèse concernant la mise en œuvre du creuset (Biringuccio, 1990).

L'indigence du mobilier et les problèmes liés à la fois à la détermination des artefacts et à l'interprétation des données archéométriques nous ont rapidement conduit à entreprendre des expérimentations. Celles-ci ont été menées en tenant compte des données archéologiques à notre disposition et des recettes fournies par les textes. Ainsi, c'est à partir des traductions française et anglaise du texte de Théophile, auteur $\mathrm{du} \mathrm{XII}^{\mathrm{e}}$ siècle, et en y associant des données venant de textes plus modernes, qu'il a été possible d'élaborer un protocole afin d'apprécier l'efficacité du procédé employant des creusets ouverts. Il ne s'agit nullement d'une étude comparative entre les différents textes mais bien le récolement de toutes les informations nécessaires au bon déroulement de ces expérimentations. En effet, le texte de Théophile ne mentionne pas certaines données et reste ambigu sur quelques points d'où l'importance de consulter d'autres auteurs. Toutefois, il est le plus ancien texte décrivant le procédé de cémentation du cuivre avec de la calamine et reste le seul à donner des informations sur le traitement minéralurgique de ce produit. On désigne sous le nom de calamine, un minerai composé de carbonate et de silicate de zinc en proportions variables. Le mot, à l'acception multiple, peut désigner l'un ou l'autre des constituants (Dieulafait, 1880). Cet élément essentiel dans la fabrication du laiton par cémentation constitue le cément. Il s'agit de la matière que l'on chauffe au contact du cuivre et qui, en se décomposant, permet la diffusion à haute température d'un ou plusieurs de ses éléments dans le métal à partir de sa surface. Afin que cette opération puisse avoir lieu, elle doit se dérouler dans un milieu réducteur qui nécessite l'usage du charbon de bois finement broyé. 
Certains auteurs préconisent également d'autres matières carbonées (Anonyme, 1747; Agricola, 2004).

\section{Description des matériauX, OBJETS ET STRUCTURES}

Pour réaliser une cémentation par creuset ouvert, il faut en premier lieu un foyer. Bien que Théophile décrive une structure métallurgique particulière que l'on peut résumer comme étant un four à sole, il a semblé préférable d'inscrire ces expérimentations dans une structure plus simple (Escalopier, 1977). Un foyer semi-enterré est utilisé (Fig. 1). Le choix de ce type de foyer doté d'une couronne réfractaire repose sur la volonté d'inscrire ce travail dans les conditions expérimentales les plus simples possibles sans présumer des difficultés induites par le choix d'une telle structure. Combustible et creuset se retrouvent donc dans un même espace d'un volume de 60 litres. La ventilation est assurée par un soufflet outre mobile que l'on positionne sur une des deux tuyères dont le foyer est équipé. Chaque pression du soufflet permet d'insuffler 6 litres d'air dans la structure. Naturellement, un point chaud se crée au débouché de la tuyère. Utilisant un seul soufflet, il apparaît un déséquilibre dans la géométrie de la température à l'intérieur du foyer. C'est dans la zone la plus chaude qu'est placé un creuset mesurant $10 \mathrm{~cm}$ de haut et $9 \mathrm{~cm}$ d'ouverture et pouvant contenir $460 \mathrm{ml}$. Cette pièce, essentielle pour l'expérimentation, est fabriquée dans une argile réfractaire très chamottée. Elle est tournée quoique les traités de métallurgie conseillent d'utiliser des creusets moulés (Ercker, 1951). En effet, les tensions induites dans la pâte par l'usage du tour fragilisent la pièce céramique. En revanche, aucun lut n'est utilisé pour protéger le creuset des variations de température d'un point à un autre de la zone de chauffe bien que l'épaisseur de la pâte du vase n'excède pas $5 \mathrm{~mm}$. Dans le creuset est

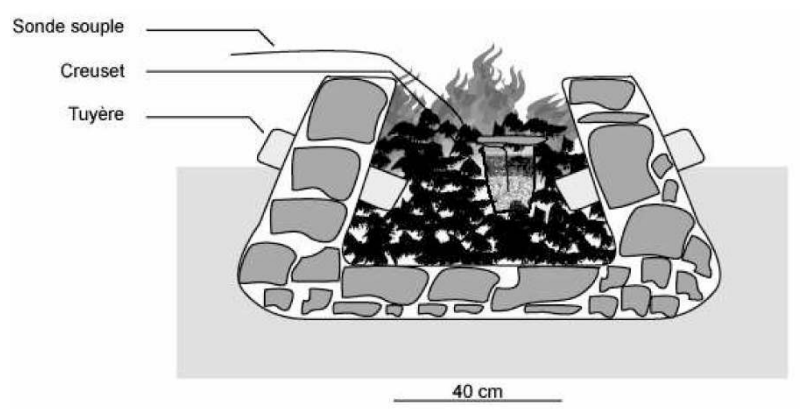

Figure 1: Coupe transversale du foyer.

Figure 1: Cross section of the hearth. inséré un thermocouple souple de type K pouvant supporter une température de $1200^{\circ} \mathrm{C}$. Il est relié à un multimètre (METRAhit 28s ${ }^{\circledR}$ ) afin de pouvoir mesurer les températures atteintes.

Dans le cadre de ces premières expérimentations, trois composés de zinc sont mis en œuvre : de l'oxyde de zinc pur issu de l'industrie chimique et à destination des céramistes, de la calamine brute et ce même minéral calciné. Ce minerai est actuellement difficile à se procurer. Son exploitation a été remplacée par celle de la blende $(\mathrm{ZnS})$ faute de gisement rentable dès la seconde moitié du XIx ${ }^{\mathrm{e}}$ siècle (Garçon, 2002). La calamine utilisée dans notre cadre expérimental provient d'un gisement de blende complètement altérée encaissé dans une formation calcaire au sein des Alpes Bergamasques. Elle a été ramassée sur la mine de Monte Trevasco exploitée au Moyen Âge (commune de Parre). Elle se compose essentiellement d'hémimorphite $\left(\mathrm{Zn}_{4}\left(\mathrm{Si}_{2} \mathrm{O}_{7}\right)(\mathrm{OH})_{2} \mathrm{H}_{2} \mathrm{O}\right)$, soit en petits cristaux blancs et limpides, soit en masses grenues de teinte grise, friables formant de petits cristaux coalescents. On note aussi la présence de smithsonite $\left(\mathrm{Zn}\left(\mathrm{CO}_{3}\right)\right)$ tenant du fer issu de la blende originelle. Le minerai est également riche en galène $(\mathrm{PbS})^{1}$. Le cuivre utilisé est un cuivre d'origine électrolytique de pureté > 99,99\% en masse. Il est exempt d'impuretés selon les normes industrielles actuelles et a fortiori selon les qualités attendues pour la période médiévale. Le métal a été façonné en tournures de longueur variable mais a une épaisseur constante de 0,45 mm. Pour le combustible comme pour la réalisation du cément, le charbon de bois provient d'une usine de carbonisation. Il s'agit uniquement de charbonnette à l'exclusion de toutes autres qualités. Enfin, détail particulier et propre aux traités de Biringuccio et d'Agricola (Biringuccio, 1990 ; MartinónTorres et Rehren, 2002), nous avons utilisé du sable pur de Fontainebleau en guise de verre moulu pour disposer dans le creuset. L'importance de cet ajout n'est pas détaillée par les auteurs mais il se retrouve dans certains ouvrages plus récents (Anonyme, 1747).

\section{Calcination de la calamine}

Le texte de Théophile rapporte que la roche extraite doit être placée sur un tas de bois puis brûlée [..., qui non confractus, sed ita ut effoditur, lignis congnestis et abundanter succensis imponitur...] (Escalopier, 1977). Nous sommes en présence d'une calcination et non d'un grillage car il s'agit bien de soumettre ce corps à une température élevée (action de calciner) et non de chauffer un minerai dans un courant

1. La description minéralogique a été faite par Pierre Rostan. 
d'air, comme s'est le cas pour les minerais sulfurés (Berthier, 1834; Braly, 1927). Théophile est le seul parmi les auteurs cités précédemment qui mentionne cette opération, à l'exception d'Ercker qui prône l'usage de la calcination tout en utilisant un composé de zinc provenant des fourneaux de réduction du minerai de plomb (Ercker, 1951). Bien que la recette de Théophile soit intéressante, les informations données restent incomplètes. Il manque notamment le temps de cette calcination. Afin de palier à ce manque, deux durées sont expérimentées : trente minutes pour l'essai 3 et douze heures pour l'essai 4.

D'après les études menées par Diffraction des Rayons $\mathrm{X}$ $(\mathrm{DRX})^{2}$, le minerai utilisé se compose principalement de calamine siliceuse hydratée (hémimorphite) et de calamine carbonatée (smithsonite) (Fig. 2). La calcination permet la transformation du carbonate de zinc (smithsonite) en oxyde de zinc par expulsion du dioxyde de carbone et la transformation de l'hydroxysilicate de zinc hydraté (hémimorphite) en silicate de zinc sous forme de willémite $\left(\mathrm{Zn}_{2}\left(\mathrm{SiO}_{4}\right)\right)$. Ces réactions se produisent à des températures de chauffe relativement faibles (inférieures à $\left.800^{\circ} \mathrm{C}\right)($ Schnabel, 1898). La recette de Théophile place la roche directement au contact du feu. Ce choix risque d'entraîner des pertes en zinc par la mise en place de réactions chimiques. En effet, dans ces conditions et pour le composé carbonaté, l'oxyde de zinc formé peut être réduit par le charbon dès $1000^{\circ} \mathrm{C}$, et ainsi libérer le zinc métal sous forme de vapeurs. Cette réduction libère aussi de l'oxyde de carbone qui peut à nouveau réduire l'oxyde de zinc en zinc métal à des températures beaucoup moins élevées (350 à $400^{\circ} \mathrm{C}$ ) (Pascal et Baud, 1932). Quant à la calamine siliceuse, la séparation du zinc et du silicate peut se faire avec du charbon à haute température (Pascal et Baud, 1932). Ainsi par ce procédé, il est fort probable que le minerai de départ perd en qualité. C'est pourquoi, dans ce cadre expérimental, le minerai de zinc est isolé du combustible pendant la calcination. Quelques gros morceaux de minerai sont placés dans un récipient, puis chauffés. La température de chauffe au niveau de la calamine est comprise entre 400 et $650^{\circ} \mathrm{C}$. Une fois la calcination terminée, la roche refroidie est broyée grossièrement afin d'être mélangée au poussier de charbon. D'après les analyses de DRX, il semblerait qu'aux températures effectuées et dès une demi-heure de chauffe, la transformation des composés de zinc se soit opérée (Fig. 2). En effet, il n'y a pas de différence significative entre l'analyse par DRX de la calamine calcinée pendant une demi-heure et celle calcinée pendant

2. Nous avons utilisé un appareil Philips X'Pert équipé d'un détecteur X'CELERATOR et d'une anticathode en cuivre permettant de produire un rayonnement $\mathrm{X}$ à angle d'incidence réglable. L'analyse s'effectue sur des pastilles de poudre. Le calibrage de l'appareillage est obtenu sur une poudre de référence (silicium).

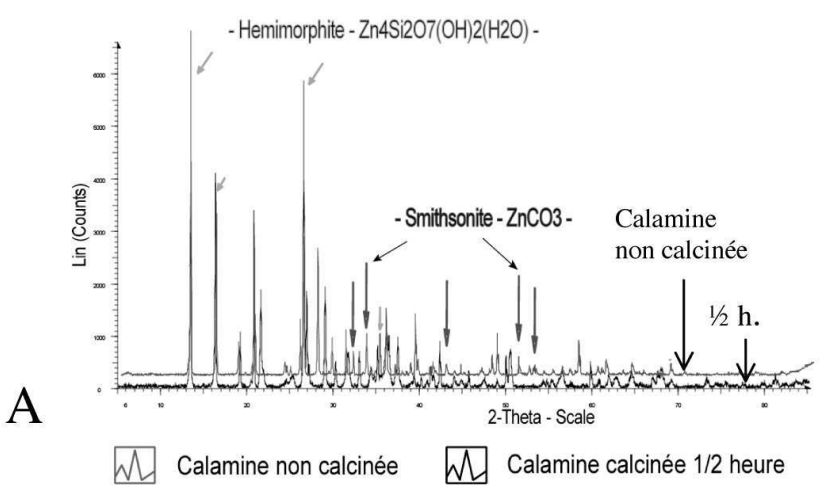

B

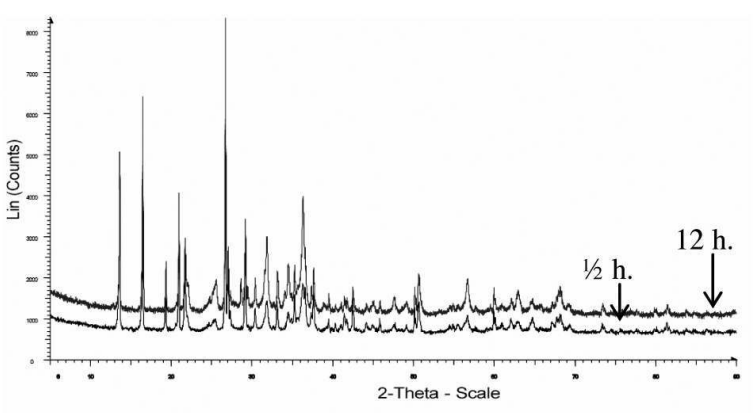

W $1 / 2$ heure de calcination Wh 12 heures de calcination

Figure 2 : Spectres de diffraction des rayons X (DRX) des minerais de zinc (A : Comparaison entre la calamine non calcinée et celle calcinée $1 / 2$ heure; $\mathrm{B}$ : Comparaison entre la calamine calcinée 1/2 heure et celle calcinée 12 heures).

Figure 2: Spectrum of diffraction $X$ of the zinc ores. (A: comparison between not calcined calamine and calcined calamine 30 minutes; B: comparison between calcined calamine 30 minutes and calcined calamine 12 hours).

douze heures. Donc il ne semble pas nécessaire de calciner guère plus de trente minutes. Afin de connaître la qualité du minerai employé, des analyses chimiques ${ }^{3}$ ont été réalisées sur des poudres présentant une granulométrie inférieure à $125 \mu \mathrm{m}$. Les concentrations en zinc varient de 40 à $50 \%$ massique pour les trois types de calamine. De plus, il n'y a pas de variation notoire pouvant être mise en relation avec les différents temps de calcination. Cette préparation du minerai n’a pas entraîné de perte sensible en zinc. On note également la présence de plomb en quantité relativement importante, entre 3 et $7 \%$ massique, et la présence de fer, autour de $2 \%$ massique. Dans la littérature, l'hémimorphite comme

3. Les analyses ont été réalisées par spectrométrie en dispersion d'énergie couplée à un microscope électronique à balayage (M.E.B., Cambridge Instruments Stereoscan) avec une tension d'accélération de $15 \mathrm{keV}$ et une durée d'analyse de 120 secondes. 
la smithsonite contiennent plus de $50 \%$ massique de zinc lorsqu'elles sont pures (Balling, 1881, Marcoux et al., 2004). Le minéral employé ici est donc d'une bonne qualité.

\section{Déroulement de l'opération DE CÉMENTATION}

$\mathrm{Au}$ total, quatre essais sont réalisés en faisant varier la nature du composé de zinc (Tableau 1). Le premier essai est fait avec l'oxyde de zinc afin de créer un essai blanc c'està-dire avec un composé chimiquement pur. Il servira par la suite de comparaison pour apprécier la qualité des laitons formés lors des trois autres essais. L'essai 2 présente un cément composé de calamine alors que les deux essais suivants se composent de calamine calcinée. L'organisation des éléments dans le creuset est celle qui est décrite dans le texte de Théophile. De bas en haut, se trouve le mélange du poussier de charbon avec de la calamine (pour les essais 2, 3 et 4) ou de l'oxyde de zinc (pour l'essai 1), suit le cuivre sous forme de tournure complètement recouvert de poussier de charbon, et pour finir, une couche de sable (Fig. 3).

La mise en route de l'opération commence par un préchauffage du creuset avec sa charge jusqu'à une température de $200{ }^{\circ} \mathrm{C}$ afin d'évacuer toute l'humidité présente (Fig. 4). Les deux heures que dure l'opération sont calculées à partir de l'introduction dans le foyer. À terme, le creuset encore chaud est retiré puis vidangé afin de recueillir l'intégralité des matériaux. Pendant la cémentation, la ventilation se fait par deux tuyères. La température est prise régulièrement. Les quatre courbes présentées à la figure 5 montrent que les conditions de chauffe sont similaires pour les quatre expérimentations. Au départ, par l'action du soufflet, la température est augmentée jusqu'à atteindre $950{ }^{\circ} \mathrm{C}$ puis est maintenue pendant soixante-quinze minutes. Ce premier plateau autour de $950{ }^{\circ} \mathrm{C}$ offre les conditions optimales pour permettre la cémentation tout en restant en dessous du point de fusion du cuivre $\left(1083^{\circ} \mathrm{C}\right)$ et au-dessus de la température d'ébullition du zinc $\left(907^{\circ} \mathrm{C}\right)$. Au bout d'une heure et quart, une augmentation de la température est recherchée à l'aide du soufflet pour atteindre les $1050{ }^{\circ} \mathrm{C}$, température qui est approximativement celle à laquelle fond un alliage $\mathrm{Cu}-\mathrm{Zn}$ contenant $10 \%$ de zinc. Cette température est maintenue pendant dix minutes (deuxième plateau) pour permettre au laiton de fondre et de former une masse. Les différents temps décrits cidessus, ont été induits par le comportement du creuset et de sa charge lors du premier essai puis répété à l'identique.

Après deux heures de cémentation, les creusets ont été retournés et vidés dans un récipient et les résultats obtenus sont peu différents d'un essai à l'autre (Fig. 6). Une première observation visuelle de l'alliage formé se présentant sous forme de masses fondues, le plus souvent sous forme de billes ou de lingotins de différentes dimensions, ne permet pas de savoir dans les deux premiers essais s'il y a eu formation de laiton. Ce n'est qu'après une abrasion de surface sur quelques billes prises au hasard, qu'il a été possible d'observer la couleur jaune caractéristique de cet alliage. Bien que lors des deux derniers essais, une coulée de laiton soit visible, de nombreuses billes conservaient une surface oxydée ne permettant pas d'apprécier la qualité du métal. Là encore, l'abrasion s'est révélée nécessaire et positive. Une deuxième remarque porte sur les masses obtenues. En comparant par simple pesée, les quantités de cuivre utilisé et d'alliages formés (Tableau 2), nous avons une augmentation qu'il est possible de traduire, tout en restant prudent, comme le pourcentage massique de zinc incorporé au cuivre initial. Ainsi l'essai 1 présenterait une concentration de $28 \%$, l'essai 2 de $14 \%$, l'essai 3 de $23 \%$ et l'essai 4 de $31 \%$ massique de zinc. Le faible pourcentage global de zinc ajouté dans le cas de l'essai 2 trouve une réponse dans l'aspect des produits métalliques. Cet essai est le seul qui présente encore des tournures en cours de fusion associées à des billes. Il s'agit de l'essai qui contenait la calamine non calcinée. Il semble bien alors que la préparation du minerai a une réelle importance pour obtenir un laiton à teneur en zinc élevée. À ce stade, il est possible d'affirmer qu'il y a bien eu formation

\begin{tabular}{|c|l|c|c|c|}
\hline \multirow{2}{*}{ ESSAI ${ }^{\circ}$} & \multicolumn{2}{|l|}{ Dénomination du composé de zinc et masse (en g) } & $\begin{array}{l}\text { Poussier de charbon } \\
\text { (masse en g) }\end{array}$ & $\begin{array}{l}\text { Tournures de } \\
\text { cuivre } \\
\text { (masse en g) }\end{array}$ \\
\hline 1 & ZnO pur & 50 & 25 & 50 \\
\hline 2 & Calamine non calcinée & 200 & 40 & 50 \\
\hline 3 & Calamine calcinée 1/2 h. & 200 & 40 & 50 \\
\hline 4 & Calamine calcinée 12 h. & 200 & 40 & 35 \\
\hline
\end{tabular}

Tableau 1 : Proportions des différentes charges utilisées pour chacune des quatre expérimentations. Table 1: Proportions of the various loads used for each test. 


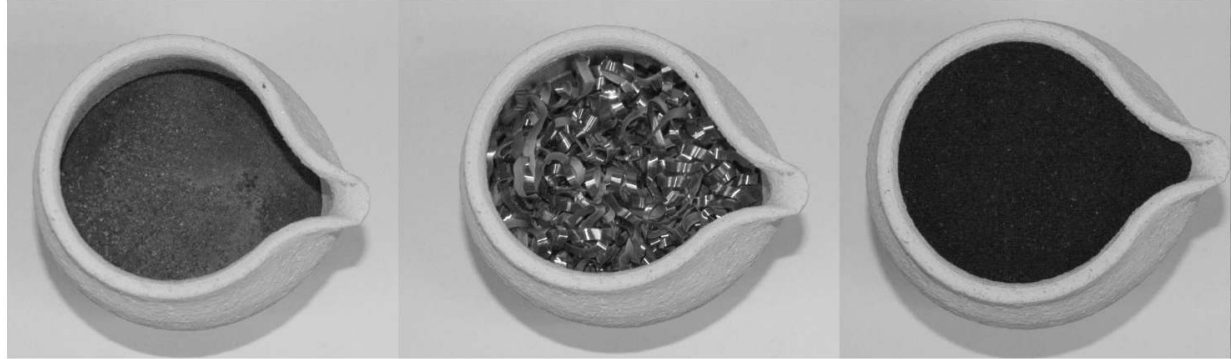

Cément
Tournures de cuivre
Poussier de charbon

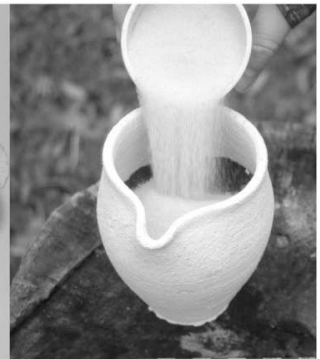

Sable

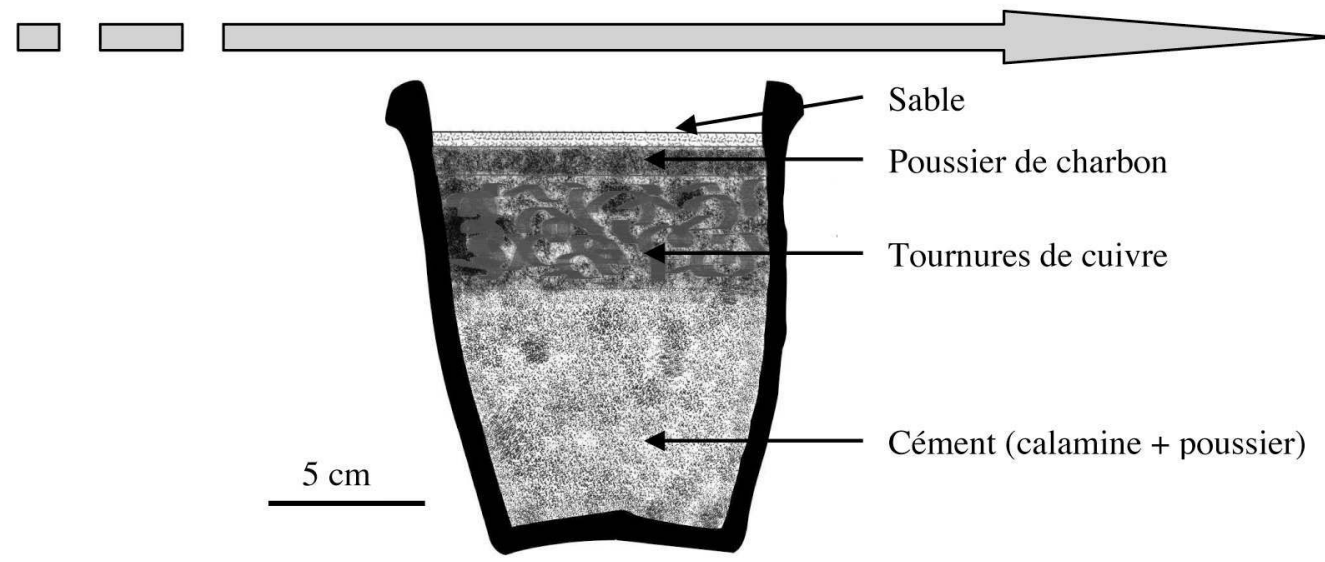

Figure 3 : Mise en place des différents éléments dans le creuset.

Figure 3: Installation of the various elements in the crucible.

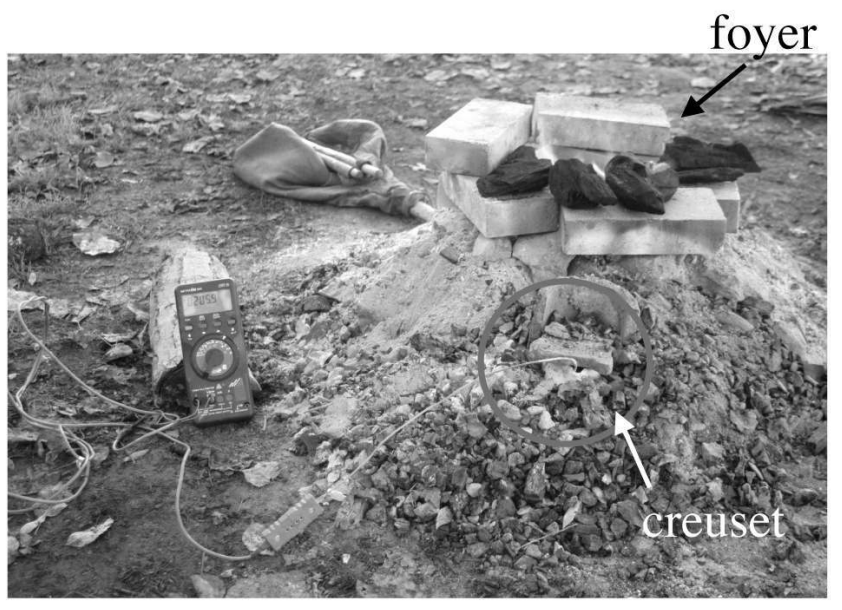

Figure 4 : Conditions expérimentales. Il s'agit du moment où le creuset est mis à préchauffer.

Figure 4: Experimental condition. It's the moment when the crucible is put to heat.

de laiton néanmoins, le recours à l'analyse chimique s'avère nécessaire pour apprécier correctement la qualité des alliages formés. Notamment, se pose le problème de l'identification des impuretés présentes dans ces métaux et provenant indéniablement du minerai de zinc.

\section{COMPOSITIONS DES LAITONS OBTENUS}

Les analyses élémentaires ${ }^{4}$ ont été effectuées sur un échantillonnage représentatif des quatre expérimentations. Quarante-cinq billes pour les quatre essais ont été sélectionnées en tenant compte de leurs différentes tailles (de 1 à $2 \mathrm{~mm}$ (A), de 2 à $5 \mathrm{~mm}$ (B), et supérieure à $5 \mathrm{~mm}(\mathrm{C})$ ). Pour le premier essai, 13 billes ont été sélectionnées, 8 pour le suivant, 11 pour le troisième et 13 pour le dernier. Les billes sont toutes préparées de la même façon. Elles sont placées dans une résine et abrasées afin d'obtenir la plus grande surface. Après avoir été polies $(1 / 4 \mu \mathrm{m})$. Le nombre de points d'analyse varie selon la dimension des échantillons analysés : 2 ou 3 pour les billes de diamètres compris entre 1 et $2 \mathrm{~mm}$, 2 à 10 pour celles entre 2 et $5 \mathrm{~mm}$, et entre 3 et 32 pour les masses de taille supérieure à $5 \mathrm{~mm}$. Les analyses montrent qu'il s'agit de laiton d'une pureté tout à fait honorable eu égard aux conditions expérimentales. Parmi les impuretés

4. Elles ont été effectuées par spectrométrie en dispersion d'énergie (système EDS, IMIX-PGT) couplé à un MEB (Hitachi S2500). La tension d'accélération utilisée est de $20 \mathrm{keV}$. Pour chaque point d'analyse d'une surface de l'ordre de $0,02 \mathrm{~mm}^{2}$, le temps d'acquisition est de $100 \mathrm{~s}$. Les analyses ont été quantifiées à partir d'étalons standards de compositions connues. L'erreur relative sur chaque élément est inférieure ou égale à $0,5 \%$ massique. 


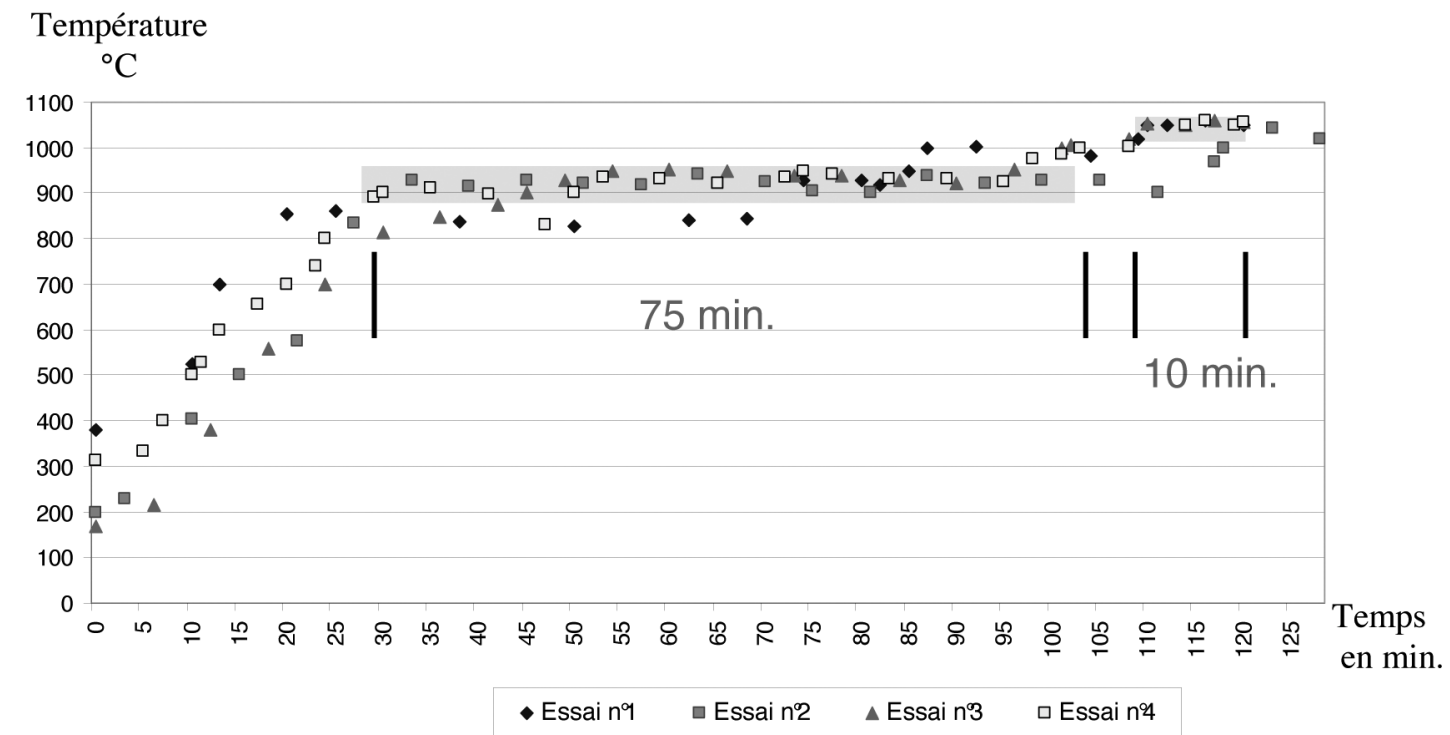

Figure 5 : Courbes des températures en fonction du temps, pour l'ensemble des essais.

Figure 5: Curves of the temperatures according to time, for the whole of the tests.

\begin{tabular}{|c|c|c|c|c|c|c|c|c|}
\hline ESSAI $n^{\circ}$ & $\begin{array}{l}\text { Dénomination du compos } \\
\text { et masse en } \mathrm{g}\end{array}$ & de zinc & $\begin{array}{l}\text { Tournures de } \\
\text { cuivre (masse } \\
\text { en g) }\end{array}$ & $\begin{array}{l}\text { Quantité théorique } \\
\text { de Zn disponible dans } \\
\text { le cément } \\
\text { (en g) } *\end{array}$ & $\begin{array}{l}\text { Masse de laiton } \\
\text { obtenue après } \\
\text { l'essai } \\
\text { (en g) }\end{array}$ & \begin{tabular}{|l} 
Estimation de la \\
masse de $\mathrm{Zn}$ \\
incorporée dans le \\
cuivre (en g)
\end{tabular} & $\begin{array}{l}\text { Zinc estimé } \\
\text { (en \% }\end{array}$ & Rendement** \\
\hline 1 & $\mathrm{ZnO}$ pur & 50 & 50 & 40,16 & 69,73 & 19,73 & 28 & $50 \%$ \\
\hline 2 & Calamine non calcinée & 200 & 50 & 106 & 58,26 & 8,3 & 14 & $7,83 \%$ \\
\hline 3 & Calamine calcinée 1/2 h. & 200 & 50 & 160,64 & 65,28 & 15,3 & 23 & $9,50 \%$ \\
\hline 4 & Calamine calcinée 12 h. & 200 & 35 & 160,64 & 50,9 & 15,9 & 31 & $9,90 \%$ \\
\hline
\end{tabular}

* La quantité théorique de $\mathrm{Zn}$ disponible dans le cément a été calculée pour l'essai 2 en tenant compte de la teneur en Zn de l'hémimorphite (54 \% mass) et de la smithsonite (52\% mass). Pour les essais 3 et 4 , on suppose que le procédé de calcination abouti à la transformation complète des composés de zinc en oxyde de zinc sans perte au feu. Dans les trois cas, il s'agit d'une quantité maximale théorique.

** Il s'agit du rapport entre la quantité de zinc incorporée et la quantité théorique de zinc disponible.

Tableau 2 : Estimation de la qualité des laitons obtenus.

Table 2: Estimate of the quality of brass obtained.

majeures, seuls le fer et le plomb ont été décelés. Néanmoins, ils restent non quantifiables précisément par la technique employée car inférieurs à 0,2\% massique dans les deux cas.

L'ensemble des concentrations en zinc, analysées par essai et par catégorie de tailles, est reporté sur la figure 7 ci-après sous forme de boîtes qui permettent pour chaque catégorie de visualiser la valeur moyenne, la médiane (le trait central) et la dispersion globale des résultats (quartiles inférieure et supérieure et les extrêmes). Conformément à ce que laissaient supposer les mesures de masse sur le terrain, il y a bien eu à chaque essai production de laiton fortement allié. La figure 7 montre aussi que les concentrations massiques en zinc des billes d'un même essai présentent une variation importante d'une masse fondue à l'autre. Par exemple pour l'essai 1, les teneurs en zinc varient de 19 à 41,5\% massique avec un écart type de 6 . Et il en va de même pour les trois autres essais où l'écart type varie de 3 à 6 . Donc pour un même essai, l'ensemble des billes de laiton a des concentrations en zinc très variables d'une bille à l'autre. De même, on n'observe pas de corrélation entre la taille des masses obtenues et leurs concentrations moyennes. Pour l'essai 1, la concentration moyenne la plus forte est obtenue pour la catégorie des billes de petites dimensions, alors que pour l'essai 4, cela est l'inverse. Il est à noter que des valeurs supérieures à $40 \%$ massique en zinc ont été obtenues pour une bille alors qu’il est souvent mentionné que ces teneurs ne dépassent pas les $30 \%$ massique. Ces résultats sont en accord avec les conclusions de J.-M. Welter (Welter, 2003). Cependant, les analyses, sur chacune des 45 


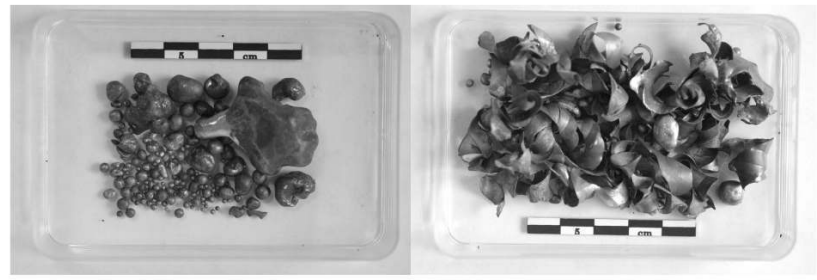

Essai $n^{\circ} 1$

Essai $n^{\circ} 2$

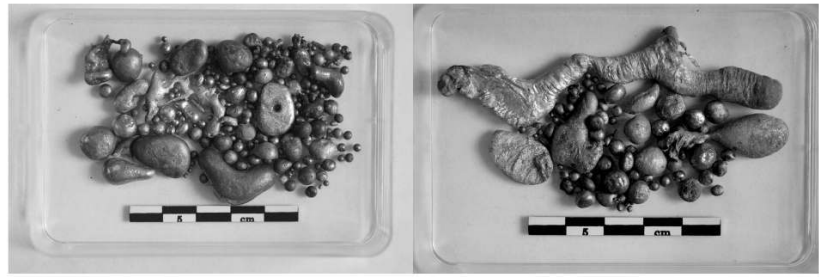

Essai $n^{\circ} 3$

Essai $n^{\circ} 4$

Figure 6 : Présentation de l'ensemble des masses métalliques obtenues après chaque cémentation.

Figure 6: Presentation of the whole of the metal masses obtained after each cementation.

billes, ont montré que 41 ont des concentrations élémentaires homogènes puisqu'elles présentent un écart type inférieur à 1 et 4 billes seulement présentent des concentrations variant d'un endroit à l'autre avec un écart type allant de 1,2 à 2,2, écarts très inférieurs à la dispersion moyenne des résultats par catégorie. En fait, une fois l'alliage $\mathrm{Cu}-\mathrm{Zn}$ formé au bout de soixante-quinze minutes, et du fait des températures maintenues à l'intérieur du creuset à environ $1050^{\circ} \mathrm{C}$, cet alliage devient très vite liquide. Cette phase va permettre de répartir de façon homogène le mélange obtenu par cémentation et n'empêche en rien la diffusion du zinc, bien au contraire, les échanges étant plus importants en phase liquide.

La figure 8 présente une comparaison des concentrations moyennes en zinc, pour chaque essai, obtenues à la fois par pesées sur le terrain et par analyses. Pour les analyses, les qualités moyennes obtenues s'échelonnent de 23 à $31 \%$ massique de zinc. La forte variation observée entre la mesure in situ à partir de la simple pesée et les dosages en laboratoire pour l'essai 2 s'explique par la stratégie d'échantillonnage. Les analyses de l'essai ne sont faites que sur des billes de laiton et ne tiennent pas compte des tournures présentant des teneurs beaucoup plus faible en zinc. De plus, la figure 8 montre que l'essai 4 a conduit à un alliage plus fortement allié. Ceci pourrait être rattaché soit à un effet bénéfique de la calcination de durée plus longue, soit à un rapport minerai-cuivre plus élevé que dans les autres essais.

Sur le terrain, par une simple pesée, il est donc possible de connaître la composition moyenne de l'alliage formé avec une très faible marge d'erreur. Cependant, une étude

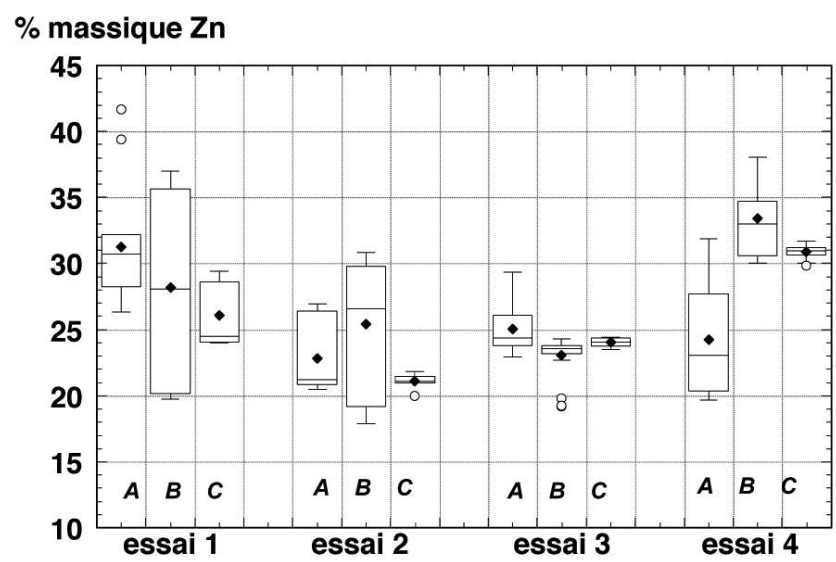

Figure 7 : Répartition de la concentration massique en zinc, pour l'ensemble des expérimentations et par catégories de tailles (A, B et $\mathrm{C}$ définis dans le texte). Les losanges correspondent à la valeur moyenne par catégorie.

Figure 7: Distribution of the zinc \%weight, for the whole of the experimentations and for the size various categories $(A, B$. C defined in the text). The rhombuses correspond to the average value by categorie.

analytique se justifie pleinement en vue de compléter les informations telle que la composition en éléments traces qu'une simple pesée ne peut donner.

\section{Conclusion et objectifs}

Les expérimentations ont montré qu'il était possible de fabriquer du laiton par cémentation à l'aide d'un creuset ouvert selon un protocole élaboré à partir de recettes anciennes. Les compositions des laitons obtenus sont comprises entre 14 et $31 \%$ massique en zinc. Indéniablement, l'emploi de creusets ouverts permet une plus grande maitrise de l'opération puisque l'on peut vérifier ce qui s'y passe par simple observation (vapeurs, couleur). Bien qu'il y ait autant de méthodes que d'auteurs, les recettes restent identiques dans les composés majeurs et dans les grandes lignes de l'organisation du processus. Toutefois, elles peuvent conduire à l'utilisation d'ingrédients qui se révèlent parfois inattendus. En effet, Agricola dans le De Natura Fossilium va jusqu'à enduire de miel des lamelles de cuivre. Faut-il y voir une particularité? Ou le miel apporte-t-il un supplément de matière carbonée? Biringuccio, quant à lui, mentionne qu'il a vu mettre en dernier lieu et recouvrant le tout, du verre moulu. Ce verre que nous avons remplacé par du sable, se retrouve très vite mélangé au reste du cément tant les turbulences sont importantes pendant la cémentation. Il en devient même gênant. Lors de la formation des billes de laiton, une multitude de grains de quartz s'incruste en surface du métal et ne pourront être évacués 


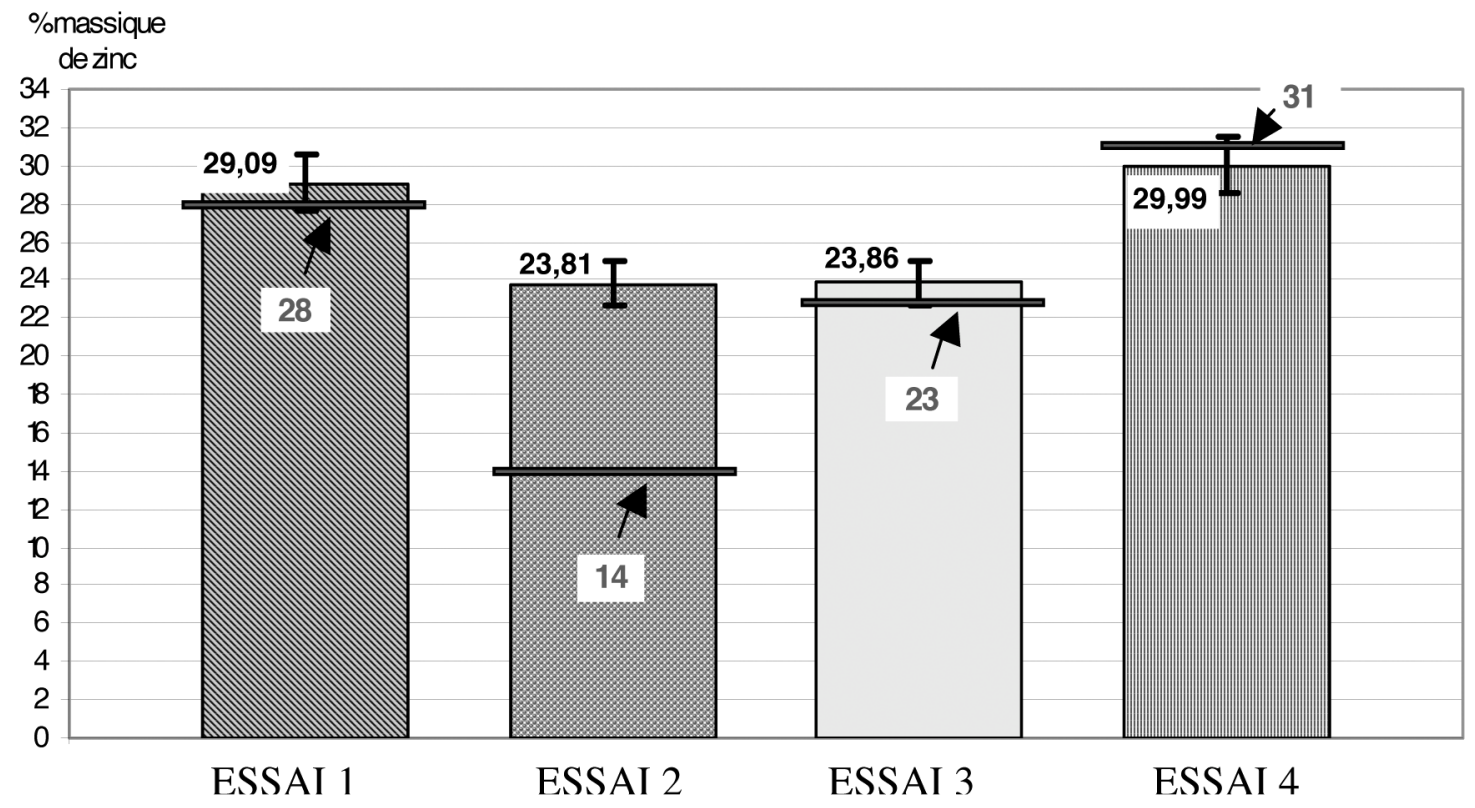

Figure 8 : Comparaison des concentrations moyennes en $\%$ massique de zinc pour chaque essai, obtenues par analyses chimiques et par pesée sur le terrain.

Figure 8: Comparison of the zinc \%weight for each test, obtained by chemical analyses and by weighing.

qu'à l'occasion d'une refonte. Cet usage du verre se retrouve mentionné par Agricola dans la seconde édition du De Natura Fossilium publiée, après sa mort, en 1558 (Martinón-Torres et Rehren, 2002). S'agit-il d'une copie des recettes des autres traités métallurgiques comme celui de Biringuccio? Ou bien est-ce un détail fourni par les métallurgistes et ajouté au même titre que les informations qui viennent enrichir cette nouvelle édition? En tout état de cause, l'usage qui a été fait du sable dans ce cadre expérimental est à proscrire.

Cette démarche va aussi permettre d'aider dans l'interprétation des vestiges liée à la fabrication du laiton. Les recherches en cours sur ces thèmes aboutissent souvent à l'intérêt qu'il y aurait à expérimenter systématiquement afin de vérifier des hypothèses (Desbat et al., 2000; Martinón-Torres et Rehren, 2002). Cette approche a l'avantage de fournir des analogues expérimentaux fabriqués dans des conditions connues et maîtrisées. Cette étude est novatrice en regard des conditions expérimentales et utilise des matériaux proches de ceux en usage pour la période considérée.

Les résultats obtenus ouvrent vers de nouveaux questionnements, notamment en vue de comprendre les phénomènes d'homogénéisation au sein d'un lingot directement coulé après une cémentation et la reproductibilité du processus. Connaissant la composition de la calamine, des études sur les problèmes de filiation pourront être menées afin de savoir quels sont les éléments traces qui se retrouvent dans le laiton après la formation de l'alliage. Et pour finir, il s'agit de reconsidérer l'utilisation des creusets ouverts retrouvés en fouille et systématiquement définis comme des creusets servant à la fonte d'alliages base cuivre.

\section{Remerciements}

Nous remercions très chaleureusement le Dr Jean-Marie Welter de la société Tréfimétaux S.A., pour les standards fournis pour l'analyse des alliages, monsieur Walter-John Chitty pour son aide lors de l'analyse par DRX et monsieur Pierre Rostan pour nous avoir fourni la calamine nécessaire à la bonne conduite de ces expérimentations et sa description minéralogique.

\section{Bibliographie}

Agricola, G., 2004. De Natura Fossilium, 1546, publié et traduit par M.-C. Bandy et J.-A. Bandy en 1955, New York, Dover phoenix editions, p. 187-188.

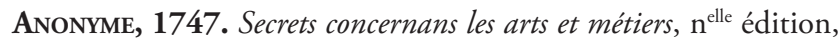
Tome 1, Bruxelles, La Compagnie, p. 14 et 26.

Balling, C., 1881. Manuel pratique de l'art de l'essayeur, guide pour l'essai des minerais, des produits métallurgiques et de combustibles, traduit par L. Gautier, Paris, Librairie F. Savy, p. 459.

ArcheoSciences, revue d'archéométrie, 30, 2006, p. 15-24 
BAYLEY, J., 1984. Roman brass-making in Britain, Journal of the Historical Metallurgy Society, 18 (1), London, Maney Publishing, p. 42-43.

BAYLEY, J., 1998. The production of brass in antiquity with particular reference to roman britain, in P. T. Craddock, 2000 years of zinc and brass, $\mathrm{n}^{\text {elle }}$ édition, London, British Museum Occasional Paper 50, p. 7-27.

Berthier, M.-P., 1834. Traité des essais par la voie sèche ou des propriétés, de la composition et de l'essai des substances, métalliques et des combustibles, Tome 1, Paris, Librairie Thomine, p. 25.

Biringuccio, V., 1572. De la Pirotechnia, 1540, traduit par V. Jaques, Paris, Chez Claude Fremy, p. 35-37.

Biringuccio, V., 1990. De la Pirotechnia, 1540, rééd., traduit par C.-S. Stanley \& M.-T. Gnudi, New York, Library of Congress Cataloging-in-Publication Data, p. 70-72.

Braly, A., 1927. Détermination et étude des minerais, Paris, Librairie Scientifique Albert Blanchard, p. 41.

Desbat, A., Meille, E., Picon, M., 2000. La préparation du laiton par cémentation à l'époque romaine, in P. Pétrequin, P. Fluzin, J. Thiriot, P. Benoit, Arts du feu et productions artisanales, $X X^{e}$ rencontres Internationales d'Archéologie et d'Histoire d'Antibes, Antibes, éd. APDCA, p. 183-188.

Dieulafait, L., 1880. Annales de Chimie et de Physique, [5], 21, Paris, Gauthiers-Villars, p. 256.

Dieulafait, L., 1880. Comptes rendus des Séances de l'Académie des Sciences, 90, Paris, Gauthiers-Villars, p. 1573.

Dumas, M., 1833. Traité de chimie appliquée aux arts, tome 4 , Paris, éd. Béchet Jeune, p. 487-488.

ERCKer, L., 1951. Treatise on ores and assaying, "Beschreibung Allerfürnemisten Mineralischen Ertzt unnd Bergkwercks arten..." ", Prague, 1574, rééd., publié et traduit par A.G. Sisco et C.-S. Stanley, Chicago, The University of Chicago Press, p. 180-181 et p. 254-257.

EsCalopier (de L'), C., 1977. Théophile Prêtre et moine, essai sur divers arts, $1^{\text {er }}$ édition 1843, Nogent-le-Roi, Librairie des Arts et Métiers, Chap. LXV, p. 224-225.

Garçon, A.-F., 2002. Un (demi) métal, 4 procédés, 2 filières, comment l'Europe inventa le zinc entre le $\mathrm{XVII}^{\mathrm{e}}$ et $\mathrm{XIX}^{\mathrm{e}}$ siècles, in Materials : research, developpement and application, Turnhout, Brepols, A. Herlea, proceedings of the XXth international congress of history of science (Liège, 20-26/07/1997), ed. H. J. Brauns, vol. XV, p. 11-29.

Hawthorne, J.-G. et Sмiтh, C.-S., 1979. On divers arts, The treatise of Theophilus, traduit par J.-G. Hawthorne et C.-S. Smith, Chicago, Dover editions, Chap. LXV, p. 143-144.

HenCKel, J.-F., 1756. Introduction à la Minéralogie, Henckelius in Mineralogia redivivus, Dresde, 1747, traduit par. G. Cavelier, tome second, Paris, p. 223-225.

Krabath, S., Lammers, D., Rehren, T. et Schneider, J., 1999. Die Herstellung und Verarbeitung von Buntmettal im karolin- gerzeitlichen Westfalen, in C. Stiegemann und M. Wemhoff, 799. Kunst und Kultur der Karolingerzeit: Karl der Große und Papst Leo III. in Paderborn; Breitag zum Katalog der Ausstellung Paderborn, Zabern, Verlag philipp von zabern, Mainz am Rhein, p. 430-437.

Le Bonniec, H., 2003. Pline l'Ancien. Histoire Naturelle, livre XXXIV, troisième tirage, traduit par $\mathrm{H}$. Le Bonniec, commenté par H. Gallet de Santerre et H. Le Bonniec, Paris, Les Belles-Lettres, p. 142 et p. 288 note $\$ 100.3$.

Macquer, P.-J., 1777. Dictionnaire de Chymie, tome I, Paris, Didot, p. 412- 420.

Marcoux, E., Bonnemaison, M., Bayle, L.-D. et Vernet, R., 2004. Miner@lia. Encyclopédie Minéralogique 3D, DVD, Deuxième édition.

Martinón-Torres, M. et Rehren, T., 2002. Agricola and Zwickau : theory and practice of Renaissance brass production in SE Germany, Journal of the Historical Metallurgy Society, 36 (2), London, Maney Publishing, p. 95-111.

Pascal, P. et Baud, P., 1931. Traité de chimie minérale, vol. 7, Paris, éd. Masson, p. 138 et 195.

Pascal, P. et Baud, P., 1934. Traité de chimie minérale, vol. 12, Paris, éd. Masson, p. 406.

Pelouze, J. et Fremy, E., 1865. Traité de chimie générale, analytique industrielle et agricole, Troisième édition, Tome 3, première partie (Chimie inorganique III, 1), Paris, Victor Masson et fils, p. 1032.

Picon, M., Le Nezet-Celestin et M., Desbat, A., 1995. Un type particulier de grands récipients en terre réfractaire utilisés pour la fabrication du laiton par cémentation, in L. Rivet (dir.), Actes du Congrès de Rouen, 25-28 mai 1995, Rouen, Société Française d'Étude de la Céramique Antique en Gaule, p. $207-$ 216.

Rehren, T., 1999. Small Size, Large scale. Roman Brass Production in Germania Inferior, Journal of Archeological science, 26, London, Academic Press, p. 1083-1087.

Rerhen, T., 2003. Crucibles as Reaction Vessels in Ancient Metallurgy, in P. Craddock et J. Lang, Mining and Metal Production - Throug The Ages, London, The British Museum Press, p. 207-215.

Schnabel, C., 1898. Traité théorique et pratique de métallurgie, traduit par L. Gautier, Paris, Librairie polytechnique Baudry et Cie, p. 19.

Welter, J.-M., 2003. The zinc content of brass : a chronological indicator?, Techne, 18, Paris/Argenton-sur-Creuse, éd. Réunion des Musées Nationaux, p. 27-36.

Wurtz, A.-D., 1873. Dictionnaire de chimie pure et appliquée contenant la chimie organique et inorganique, la chimie appliquée à l'industrie, à l'agriculture et aux arts, la chimie analytique, la chimie physique et la minéralogie, Tome second, première partie, Paris, Librairie Hachette et Cie, p. 203. 